POS PROCEEDINGS

\title{
Azimuthal HBT and transverse momentum fluctuations from CERES
}

Dariusz Miśkowiec ${ }^{c}$ for the CERES Collaboration: D. Adamováa ${ }^{\text {, G. Agakichiev }}{ }^{\mathrm{b}}$, A. Andronic c , D. Antończyk ${ }^{\mathrm{d}}$, H. Appelshäuser ${ }^{\mathrm{d}}$, V. Belaga ${ }^{\mathrm{b}}$, J. Bielčíkováe, ${ }^{\mathrm{f}}$, P. Braun-Munzinger ${ }^{c}$, O. Busch ${ }^{c}$, A. Cherling, S. Damjanović ${ }^{f}$, T. Dietel $^{\mathrm{h}}$, L. Dietrich ${ }^{\mathrm{f}}$, A. Drees ${ }^{i}$, W. Dubitzky ${ }^{\text {f }}$, S. I. Esumi ${ }^{\mathrm{f}}$, K. Filimonov ${ }^{\mathrm{f}}$, K. Fomenko ${ }^{\mathrm{b}}$, Z. Fraenkel ${ }^{\mathrm{g}}$, C. Garabatos $^{c}$, P. Glässel ${ }^{\mathrm{f}}$, G. Hering $^{\mathrm{c}}$, J. Holeczek $^{\mathrm{c}}$, M. Kalisky $^{\mathrm{c}}$, S. Kniege ${ }^{\mathrm{d}}$, V. Kushpil ${ }^{\mathrm{a}}$, A. Maas ${ }^{\mathrm{c}}$, A. Marín ${ }^{\mathrm{c}}$, J. Miloševićf ${ }^{\mathrm{f}}$ D. Miśkowiec ${ }^{\mathrm{c}}$, R. Ortega ${ }^{\mathrm{f}}$, Y. Panebrattsev ${ }^{\mathrm{b}}$, O. Petchenova ${ }^{\mathrm{b}}$, V. Petráček ${ }^{\mathrm{f}}$, M. Płoskońd $^{\mathrm{d}}$, S. Radomski ${ }^{\mathrm{f}}$, J. Rak ${ }^{\mathrm{c}}$, I. Ravinovich ${ }^{\mathrm{g}}$, P. Rehak', H. Sako ${ }^{\mathrm{c}}$, W. Schmitz ${ }^{\mathrm{f}}$, S. Schuchmann ${ }^{\mathrm{d}}$, J. Schukraft ${ }^{\mathrm{k}}$, S. Sedykh ${ }^{c}$, S. Shimansky ${ }^{\text {}}$, R. Soualah ${ }^{\mathrm{f}}$, J. Stachel $^{\mathrm{f}}$, M. Šumbera ${ }^{\mathrm{a}}$, H. Tilsner $^{\mathrm{f}}$, I. Tserruya ${ }^{\mathrm{g}}$, G. Tsiledakis ${ }^{\mathrm{c}}$, J. P. Wessels ${ }^{\mathrm{h}}$, T. Wienold ${ }^{\mathrm{f}}$, J. P. Wurm ${ }^{\mathrm{e}}$, S. Yurevich ${ }^{\mathrm{c}}$, V. Yurevich ${ }^{\mathrm{b}}$

a NPI ASCR, Řež, Czech Republic

b JINR Dubna, Russia

${ }^{\mathrm{c}}$ GSI Darmstadt, Germany

${ }^{\mathrm{d}}$ Frankfurt University, Germany

${ }^{\mathrm{e}} \mathrm{MPI}$, Heidelberg, Germany

${ }^{\mathrm{f}}$ Heidelberg University, Germany

${ }^{\mathrm{g}}$ Weizmann Institute, Rehovot, Israel

${ }^{\mathrm{h}}$ Münster University, Germany

${ }^{\mathrm{i}}$ SUNY Stony Brook, U.S.A.

$\mathrm{j}_{B N L}$, Upton, U.S.A.

${ }^{\mathrm{k}}$ CERN, Geneva, Switzerland

E-mail: d.miskowieclgsi.de

\begin{abstract}
I present here preliminary results of analysis of the data collected by CERES in a $\mathrm{Pb}+\mathrm{Au}$ collision experiment at the full SPS energy. The focus is on two particular hadronic observables: azimuthal Hanbury-Brown Twiss correlations between pions and fluctuations of the transverse momenta of charged particles.
\end{abstract}

Critical Point and Onset of Deconfinement - 4th International Workshop

July 9 - 13, 2007

Darmstadt, Germany 


\section{Introduction}

CERES is a dilepton experiment at the CERN SPS, known for its observation of enhanced production of low mass $\mathrm{e}^{+} \mathrm{e}^{-}$pairs in collisions between heavy nuclei [1]. The upgrade of CERES in 1997-1998 by a radial Time Projection Chamber (TPC) [2] allowed to improve the momentum resolution and the particle identification capability while retaining the cylindrical symmetry. The upgraded experiment is shown in Fig. 1. The upgrade also extended the sensitivity of CERES to hadrons and made possible results like those described below. The measurement of central $\mathrm{Pb}+\mathrm{Au}$ collisions at the maximum SPS energy of $158 \mathrm{GeV}$ per nucleon in the fall of 2000 was the first run of the fully upgraded CERES and at the same time the last run of this experiment. About 30 million $\mathrm{Pb}+\mathrm{Au}$ collision events at $158 \mathrm{GeV}$ per nucleon were collected, most of them with centrality within the top $7 \%$ of the geometrical cross section $\sigma_{\mathrm{G}}=6.94 \mathrm{~b}$. Small samples of the $20 \%$ and the minimum bias collisions, as well as a short run at $80 \mathrm{AGeV}$, were recorded in addition. The dilepton mass spectra from this experiment were published in [3]. In this talk I present two particular results of hadron analysis, the azimuthal dependence of two-pion correlations and a differential $p_{t}$ fluctuation study.

\section{Azimuthal HBT}

The two-pion correlation analysis was performed in the longitudinally co-moving frame (LCMS) defined by the vanishing $z$ component of the pair momentum ( $z$ axis is parallel to the beam). The momentum difference in this frame, $\mathbf{q}=\mathbf{p}_{2}-\mathbf{p}_{1}$, was decomposed into the "out", "side", and "long"

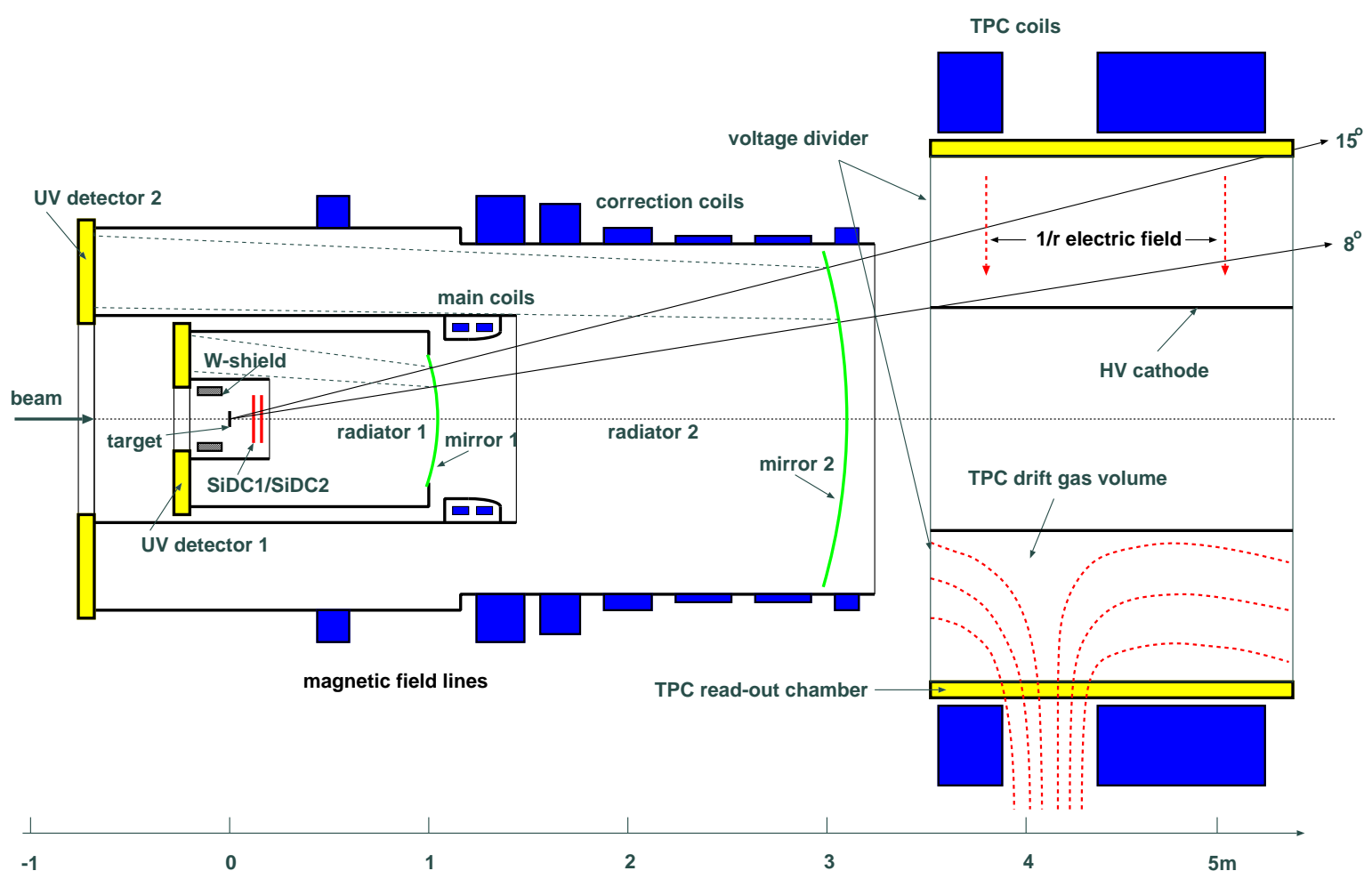

Figure 1: Upgraded CERES setup in 2000. The apparatus has a cylindrical symmetry. The results presented here are based on the tracks measured in the Time Projection Chamber (TPC). 

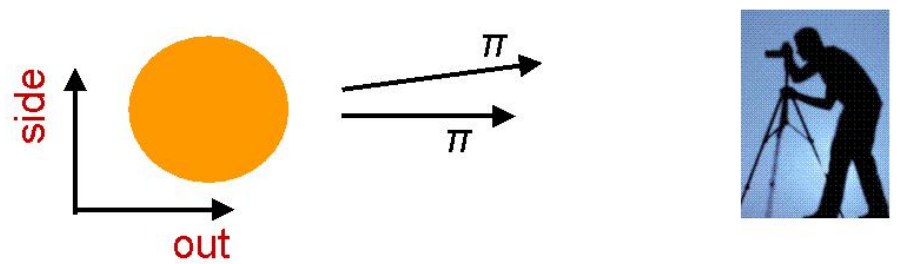

Figure 2: Definition of the transverse Bertsch-Pratt axes: out is parallel and side perpendicular to the transverse momentum of the pion pair; both are perpendicular to the beam axis.

components following the Bertsch-Pratt convention, with $q_{\text {long }}$ pointing along the beam and $q_{\text {out }}$ along the pair transverse momentum (Fig. 2). The $\pi^{-} \pi^{-}$and $\pi^{+} \pi^{+}$correlation functions, defined as the three-dimensional $\mathbf{q}$ distributions of pion pairs from the same event, divided by the analogous distributions of pairs constructed from different events (event mixing), were fitted by

$$
C_{2}(\mathbf{q})=N \cdot\left\{(1-\lambda)+\lambda \cdot F_{c}\left(q_{i n v}\right)\left[1+\exp \left(-\sum_{i, j=1}^{3} R_{i j}^{2} q_{i} q_{j}\right)\right]\right\} .
$$

The normalization factor $N$ is needed because the number of pairs from event mixing is arbitrary. The correlation strength $\lambda<1$ reflects the tails of the source distribution caused by the pions from long-lived resonances, the finite q-resolution, and the contamination of the pion sample by other particle species. The $R_{i j}^{2}$ fit parameters, with the indices $i, j$ being \{out, side, long\}, are related to the size of the source emitting pions of given momentum [4] and are therefore called source radii. The $F_{c}\left(q_{i n v}\right)$ factor, $q_{i n v}=\sqrt{-\left(p_{2}^{\mu}-p_{1}^{\mu}\right)^{2}}$, accounts for the mutual Coulomb interaction between the pions and was calculated by averaging the nonrelativistic Coulomb wave function squared over a realistic source size. The Coulomb factor was attenuated by $\lambda$ similarly as the rest of the correlation function peak; the importance of this was demonstrated in [5]. The fits were performed by the minimum negative loglikelihood method with the Poissonian number of true pairs. The source radii obtained from the fit were corrected for the finite momentum resolution.
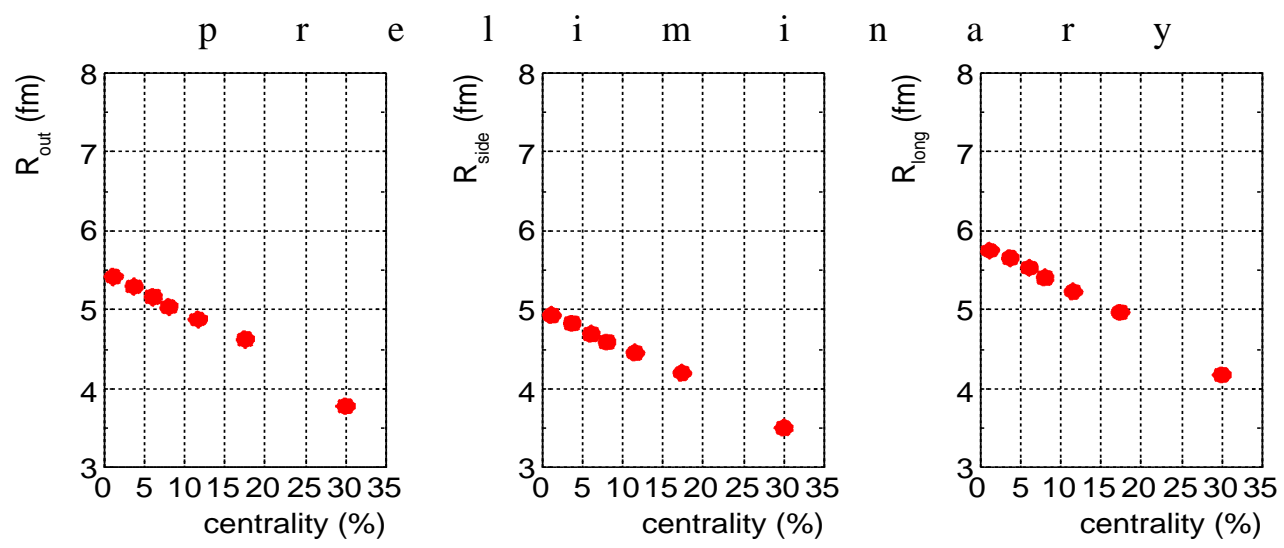

Figure 3: Centrality dependence of the pion source radii. The mean pion transverse momentum is $0.47 \mathrm{GeV} / \mathrm{c}$. The data are preliminary and come from central $\mathrm{Pb}+\mathrm{Au}$ collisions at $158 \mathrm{GeV}$ per nucleon. 
The correction was determined by Monte Carlo and was rather insignificant for $R_{\text {side }}$ and $R_{\text {long }}$; for $R_{\text {out }}$ it depends on the pair $p_{t}$ and can be as large as $20 \%$. The source radii obtained for different centralities are shown in Fig. 3. Like in the first CERES HBT analysis [5] and in numerous other studies the radii roughly scale with the cube root of particle multiplicity.

With the access to the source size provided by the two-pion correlations it is interesting to look for a possible anisotropy of the pion source in the transverse plane. The fireball created in a nuclear collision with a finite impact parameter is initially elongated in the direction perpendicular to the reaction plane. In the course of transverse expansion, with the pressure gradient larger in-plane than out-of-plane, the initial asymmetry should get reduced or even reversed. A dependence of the pion source radius on the pion azimuthal emission angle with respect to the reaction plane would be a signature of the source eccentricity at the decoupling time (Fig. 4).

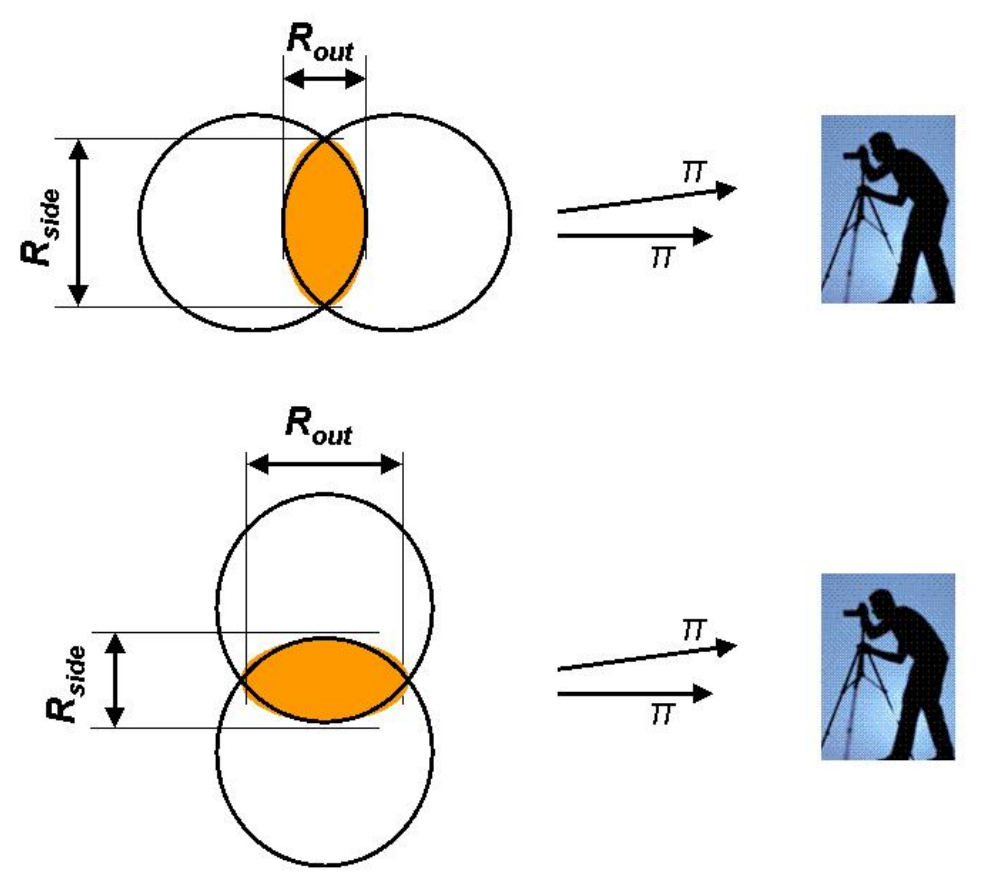

Figure 4: Initial transverse anisotropy of the fireball, and how it should manifest itself via the pion source radii. The top and bottom panels represent the pion emission in-plane and out-of-plane, respectively. The $R_{\text {out }}$ radius should be smallest for pions emitted in-plane and largest for those out-of-plane, and the $R_{\text {side }}$ radius should have the opposite behavior - unless the initial anisotropy gets reduced or reversed during the transverse expansion.

Before one can sort pions according to their azimuthal angle with respect to the reaction plane the latter has to be determined. The azimuthal angle of the reaction plane was estimated for each event via the preferred direction of the particle emission aka elliptic flow. The particles were weighted with their transverse momentum:

$$
\begin{aligned}
Q_{2}^{X} & =\sum_{i} p_{t} \cos \left(2 \phi_{i}\right) \\
Q_{2}^{Y} & =\sum_{i} p_{t} \sin \left(2 \phi_{i}\right) .
\end{aligned}
$$




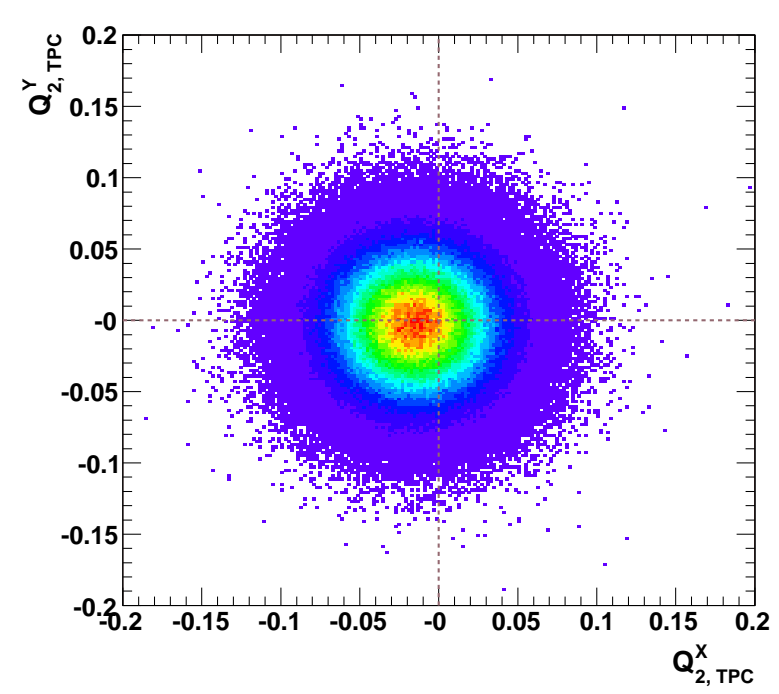

Figure 5: The raw distribution of the $\left(Q_{2}^{X}, Q_{2}^{Y}\right)$ vector used to determine the azimuthal orientation of the reaction plane. The distribution was recentered and made round run-by-run in order to make the resulting distribution of the reaction plane angle uniform.

The raw distribution $n\left(Q_{2}^{X}, Q_{2}^{Y}\right)$ is shown in Fig. 5. The distribution was recentered and made round run-by-run in order to make the distribution of the reconstructed reaction plane angle uniform. The reaction plane angle was calculated (modulo $\pi$ ) from the calibrated $Q_{2}$ components via

$$
\Phi_{\mathrm{RP}}=\frac{1}{2} \arctan \left[\frac{Q_{2}^{Y}}{Q_{2}^{X}}\right] .
$$

The resolution of the reaction plane angle, estimated via the subevevent method, was 31-38 .

With the event plane known for each event the pion pairs were sorted into 8 bins covering $(-\pi / 2, \pi / 2)$ according to their azimuthal angle with respect to the reaction plane $\Phi^{*}=\Phi_{\text {pair }}-$ $\Psi_{\mathrm{RP}}$. During event mixing it was required that the two events had similar reaction plane angles: $\left|\Psi_{\mathrm{RP}}^{2}-\Psi_{\mathrm{RP}}^{1}\right|<7.5^{\circ}$. The eight correlation functions were fitted by Eq. 2.1 ; the resulting $R_{\text {out }}, R_{\text {side }}$, $R_{\text {long }}$, and the cross-terms are shown in Fig. 6 . In order to quantify the anisotropies visible there the squared source radii were then fitted with

$$
R_{i}^{2}=R_{i, 0}^{2}+2 R_{i, 2}^{2} \cos \left(2 \Phi^{*}\right)
$$

with $i$ denoting \{out,side,long\}. The out-side cross-term was fitted by a similar formula but with sine rather than cosine and requesting that $R_{o s, 0}=0$. The $R_{i, 0}$ 's obtained coincide with the mean radii presented in Fig. 3. The second Fourier components $R_{i, 2}$ 's, which represent the eccentricity of the observed pion source, are plotted in Fig. 7 along with the analogous results obtained at the AGS [6] and at RHIC [7, 8]. The values of $R_{\mathrm{out}, 2}^{2}$ and $R_{\mathrm{os}, 2}^{2}$, consistent in sign and magnitude, indicate a pion source elongated out-of-plane. The $R_{\text {side }}$ anisotropy, on the other hand, is close to zero. In particular, it seems to be significantly reduced when going from AGS to SPS energies. The fact that anisotropies in $R_{\text {side }}$ and, especially, $R_{\text {long }}$ do not vanish in the limit of central collisions is unexpected and is still under investigation. 

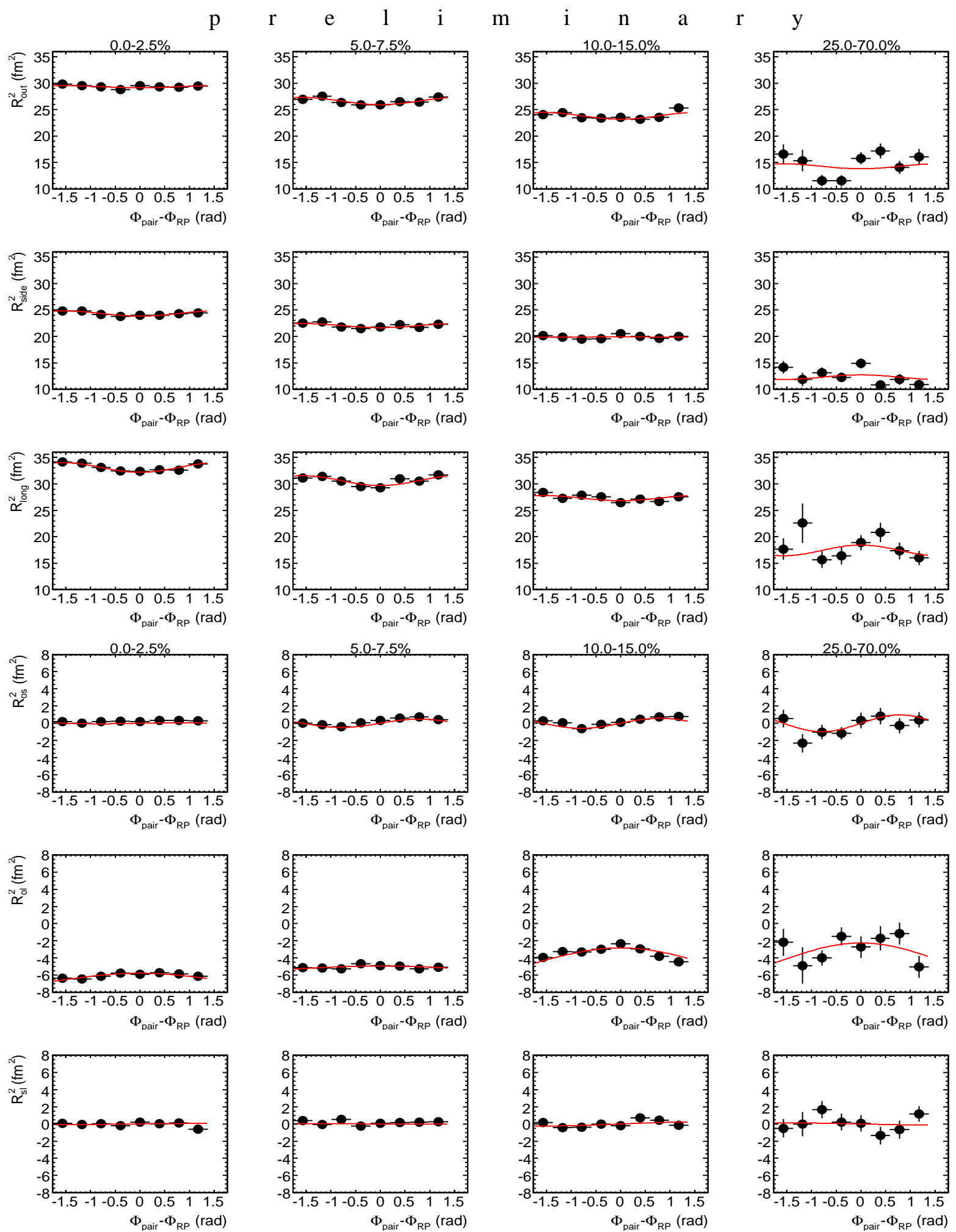

Figure 6: Pion source radii squared vs. the pion azimuthal emission angle with respect to the reaction plane $\Phi^{*}=\Phi_{\text {pair }}-\Psi_{\mathrm{RP}}$. The columns represent four of the seven analyzed centralities. Oscillations indicate pion source anisotropy. The data are preliminary and come from central $\mathrm{Pb}+\mathrm{Au}$ collisions at $158 \mathrm{GeV}$ per nucleon. Lines represent results of the Fourier decomposition (2.5). 

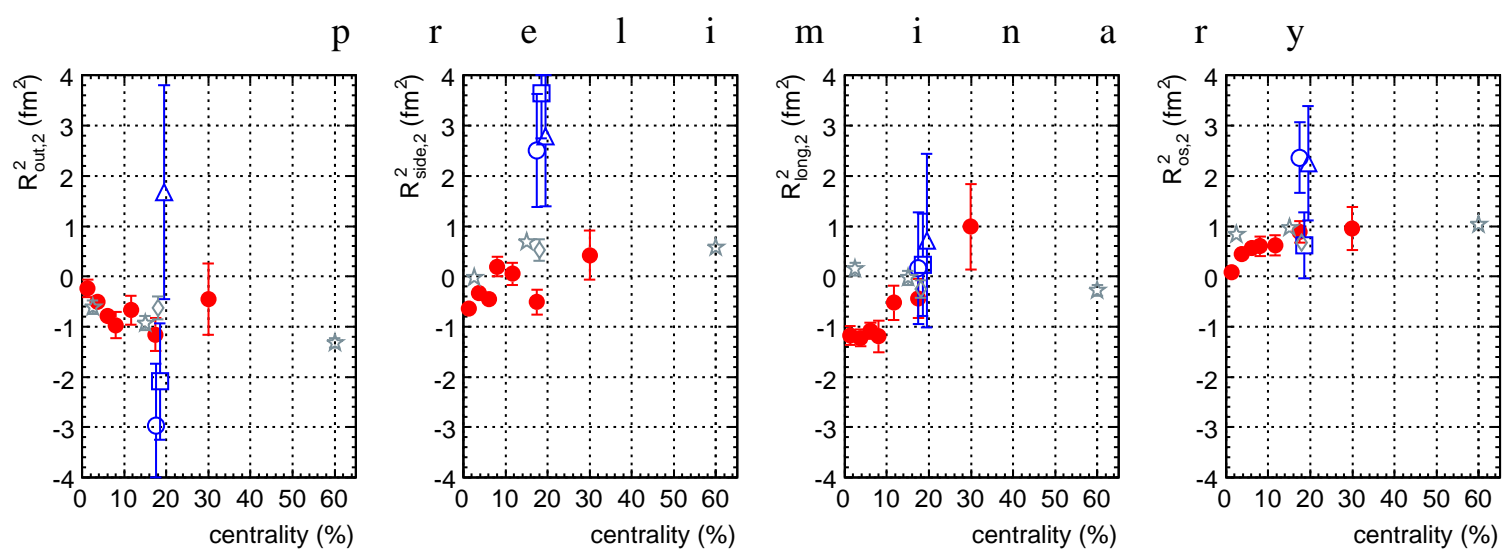

Figure 7: Azimuthal pion source eccentricity, represented by the second Fourier component of radii squared $R_{i}^{2}\left(\Phi^{*}\right)$ (full symbols). The data are preliminary and come from central $\mathrm{Pb}+\mathrm{Au}$ collisions at $158 \mathrm{GeV}$ per nucleon. Positive and negative pion pairs have been combined. The mean pion transverse momentum is $0.47 \mathrm{GeV} / \mathrm{c}$. The results are to be compared to the analogous measurements at the AGS (open blue symbols) and RHIC (rhombus and stars for 130 and $200 \mathrm{GeV}$, respectively).

\section{Transverse momentum fluctuations}

It is expected that event-by-event fluctuations are enhanced in the vicinity of the QCD critical point [9]. The first CERES results indicated that over-statistical $p_{t}$ fluctuations exist in heavyion collisions and that their magnitude is independent of the collision energy within the SPS and RHIC range (left panel of Fig. 8). This independence of collision energy is to be contrasted with a non-monotonous centrality dependence (right panel of the same figure).

In order to understand the origin of the fluctuations a differential analysis of $p_{t}$ correlations was performed in [11]. The covariance between transverse momenta of charged particles was studied there as a function of the pair opening angle in azimuth $\Delta \phi$ and in pseudorapidity $\Delta \eta$ (Fig. 9). This representation allows to identify immediately at least three components contributing
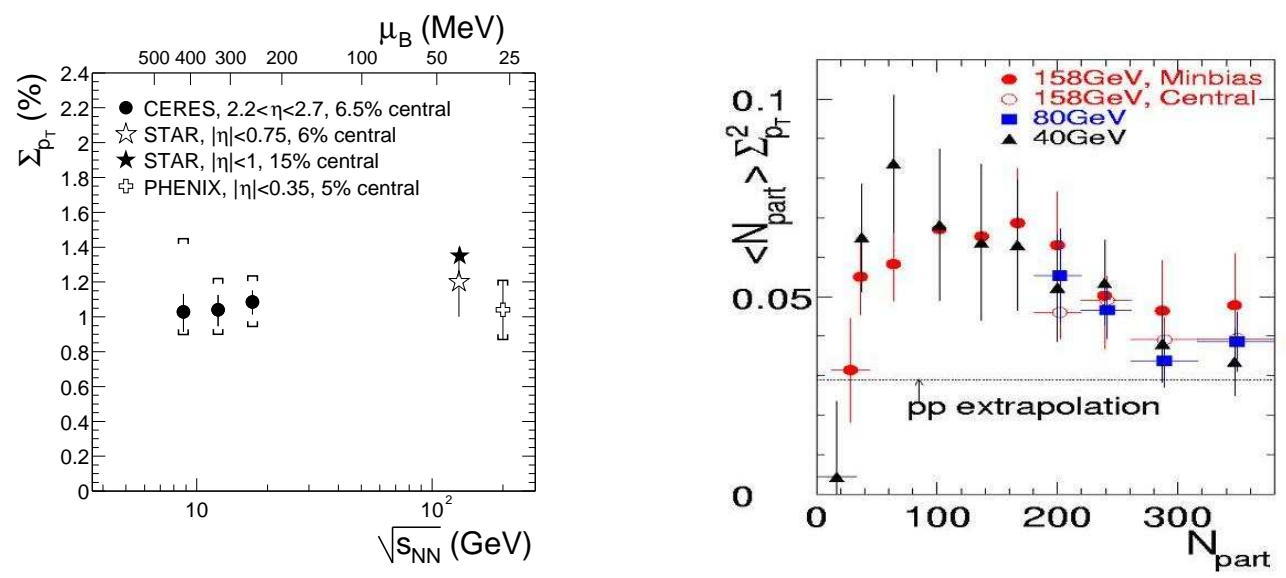

Figure 8: Left: beam energy dependence (or rather independence) of $p_{t}$ fluctuations. Right: nonmonotonous centrality dependence of the same quantity. Both figures are taken from [10]. 


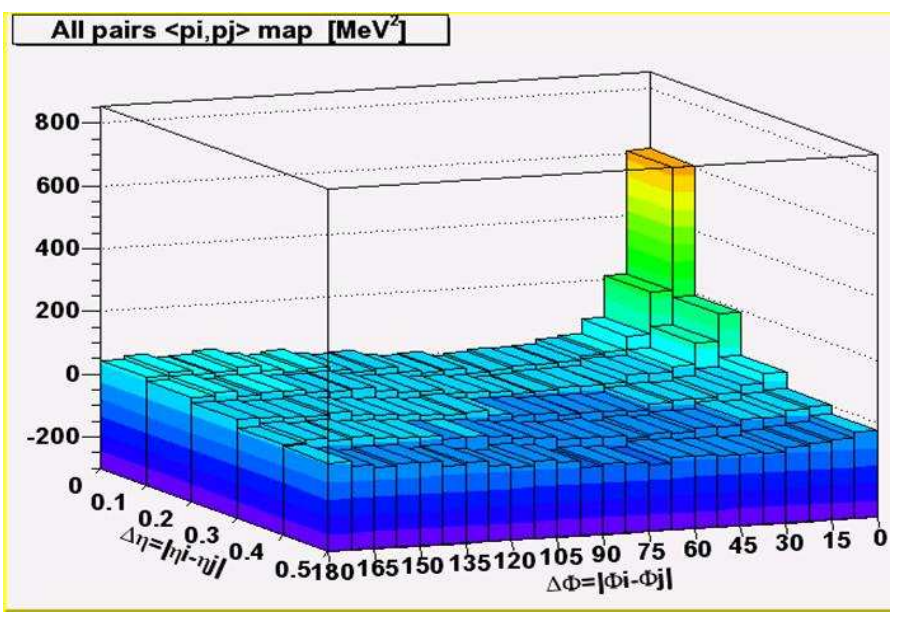

Figure 9: Transverse momentum covariance as a function of the pair opening angle. Short and long range correlations are visible. The plot is taken from [11].
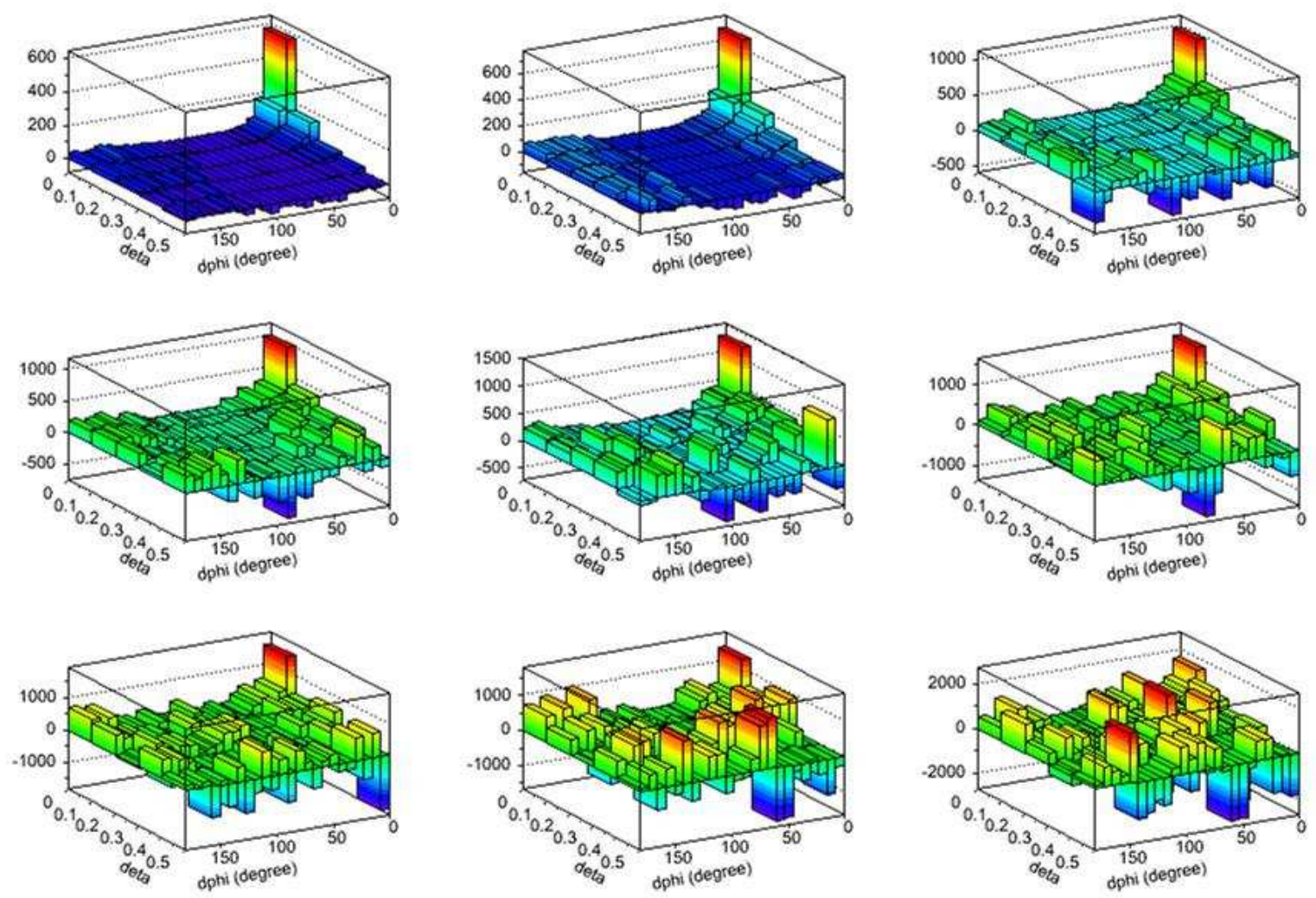

Figure 10: Differential transverse momentum covariance for centralities 0-10\% (top left), 10-20\%, ..., 80$90 \%$ (bottom right). The two most central bins have highest statistics because most of the data were taken with a central trigger. Events with centralities above $60 \%$ may be contaminated by non-target interactions. The data are preliminary and come from central $\mathrm{Pb}+\mathrm{Au}$ collisions at $158 \mathrm{GeV}$ per nucleon. 
to the effect: 1) short range correlations (quantum statistics, Coulomb, jet fragmentation) at small opening angles; 2) away-side correlations (dijets) peaked at $\Delta \phi=180^{\circ}$; 3) elliptic flow, introducing a $\cos (2 \Delta \phi)$ oscillation. Repeating the same analysis for bins of centrality (Fig. 10) and selecting different $(\Delta \phi, \Delta \eta)$ regions one can now study separately the centrality dependence of the individual components. The result is shown in Fig. 11. The short range and away-side correlation regions have a centrality dependence similar to the inclusive $p_{t}$ fluctuation. This is a natural consequence of the fact that their contributions are dominant. Apparently, the most interesting region is the one around $\Delta \phi=45^{\circ}$, free of the influence of the short range and away-side correlations and where the elliptic flow vanishes, and therefore ideal for the critical point search. The $p_{t}$ covariance for these track pairs is close to zero and shows no centrality dependence within the statistical errors.

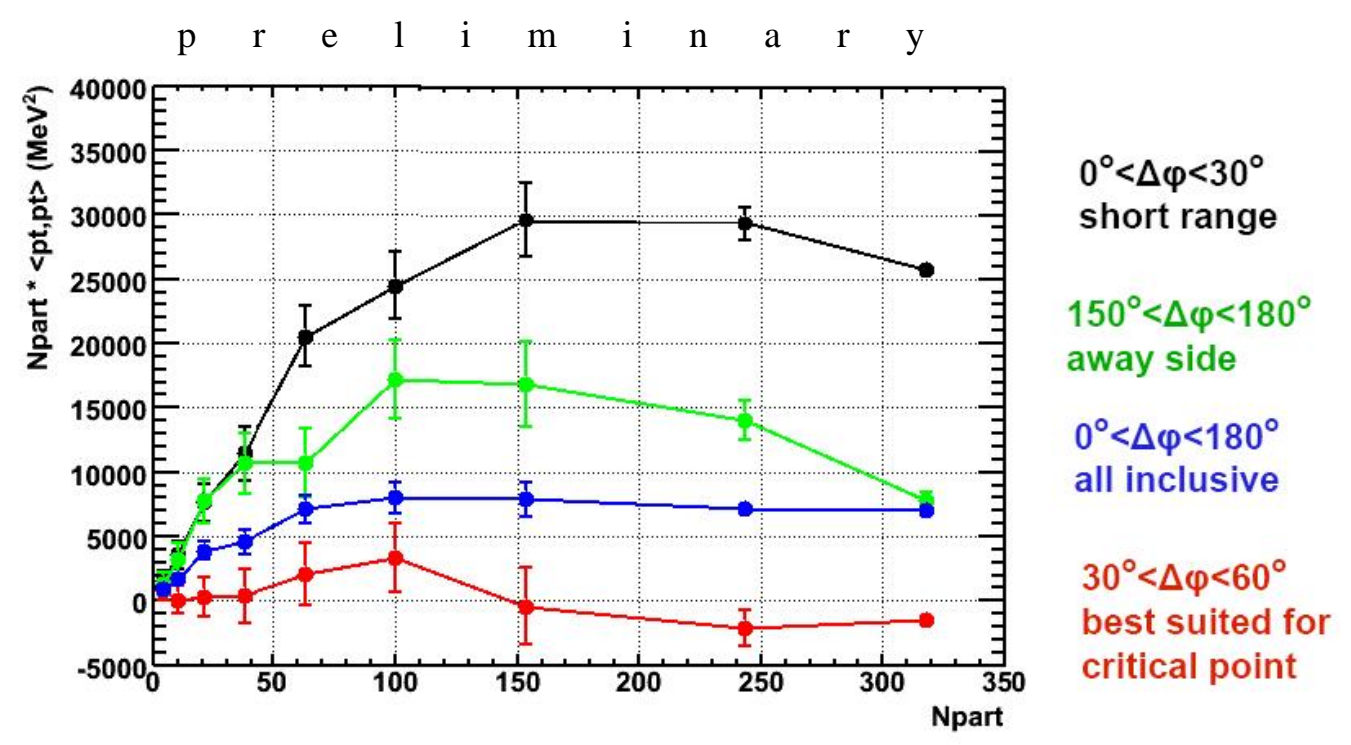

Figure 11: Centrality dependence of $p_{t}$ covariance of track pairs with different azimuthal opening angles. The data are preliminary and come from central $\mathrm{Pb}+\mathrm{Au}$ collisions at $158 \mathrm{GeV}$ per nucleon. The covariance has been multiplied with the number of participants; in this representation the plots can be compared to the inclusive analysis in the right panel of Fig. 8. The connecting lines are to guide the eye.

Results of a similar analysis applied for the first time to $80 \mathrm{GeV}$ data are shown in Fig. 12. Also here the $\Delta \phi=45^{\circ}$ component seems to be centrality independent within the errors. In order to study the collision energy dependence, in Fig. 13 the $p_{t}$ fluctuations from events with centrality of $0-10 \%$ are plotted along with RHIC results [12]. While the inclusive (all opening angles) data confirm the previous observation that the $p_{t}$ fluctuations are positive and independent of the collision energy the analogous fluctuations restricted to the pairs with the azimuthal opening angles between 30 and $60^{\circ}$, for which the "trivial contributions" are suppressed and which thus are ideal for the critical point search, give an entirely different picture. There, the fluctuations are negligible at 158 and slightly negative at $80 \mathrm{AGeV}$. (A quantitative study would be needed to judge whether or not such a negative value might be resulting from momentum conservation.) The data show no indication of the critical point fluctuations. 


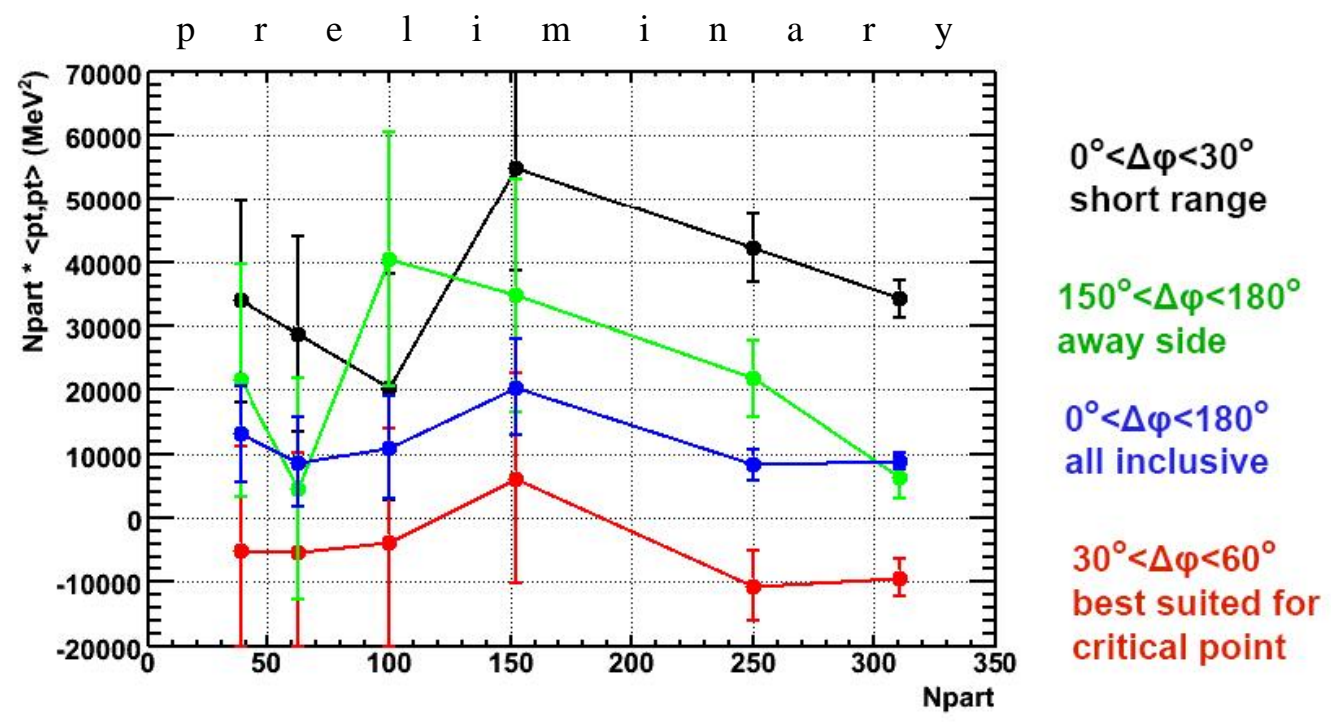

Figure 12: Same as previous figure but for $\mathrm{Pb}+\mathrm{Au}$ collisions at $80 \mathrm{GeV}$ per nucleon.

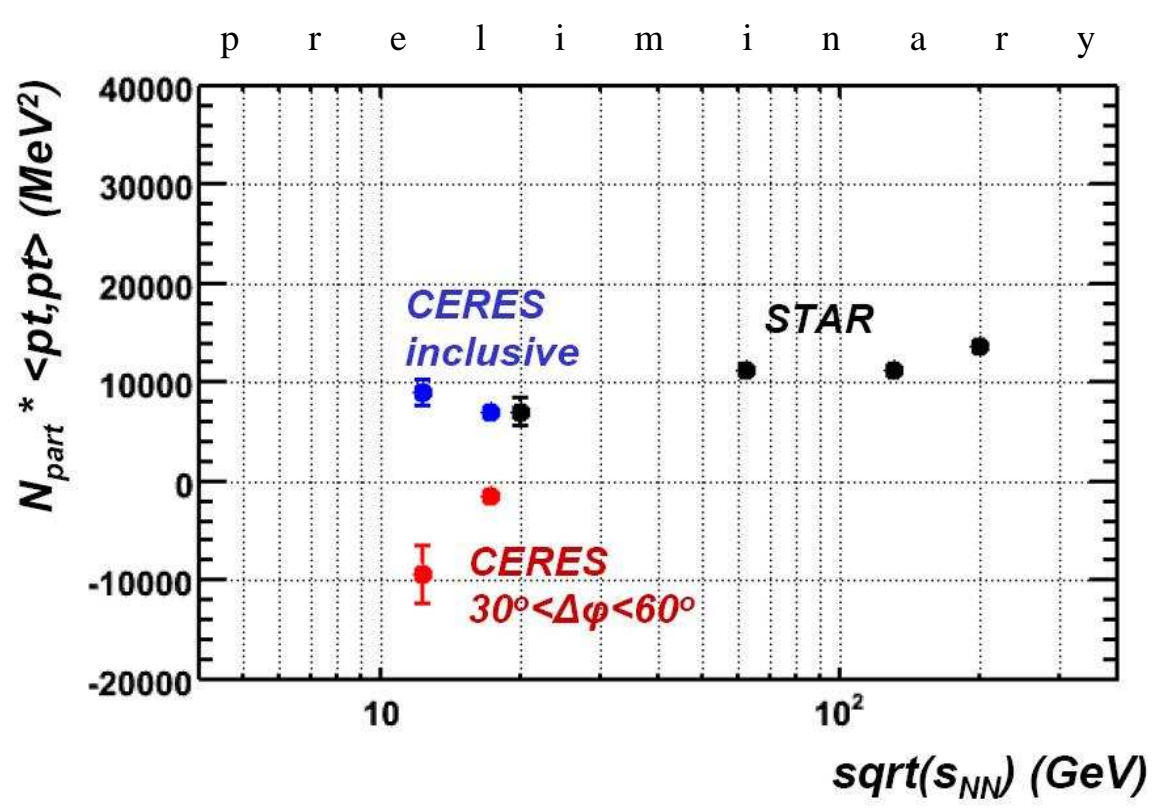

Figure 13: Transverse momentum fluctuations in central $0-10 \% \mathrm{~Pb}+\mathrm{Au}$ or $\mathrm{Au}+\mathrm{Au}$ collisions as a function of the collision energy. The blue and black points represent the inclusive (all opening angles) data from CERES and STAR, respectively. The two red points are from CERES and were obtained using track pairs with azimuthal opening angles of $30-60^{\circ}$ for which "trivial" contributions are suppressed and which therefore are ideal to look for the critical point fluctuations. The CERES data are preliminary and come from central $\mathrm{Pb}+\mathrm{Au}$ collisions at 80 and $158 \mathrm{GeV}$ per nucleon. 


\section{Summary}

Two particular hadronic results of the last CERES $\mathrm{Pb}+\mathrm{Au}$ experiment, the azimuthal pion HBT and the transverse momentum fluctuation analysis, were presented. The HBT analysis yields a moderate out-of-plane elongation of the source, rather similar to the one observed at the AGS and at RHIC, except when looking in the direction perpendicular to the beam and to the pion $p_{t}$ in which case the anisotropy seems to be greatly reduced when going from the AGS to the SPS. Transverse momentum fluctuations become much more sensitive to the critical point when the analysis is restricted to pairs of tracks emitted at approximately $45^{\circ}$ to each other in azimuth; no indication of critical fluctuations is seen in the data analyzed this way.

\section{Acknowledgments}

The CERES collaboration acknowledges the good performance of the CERN PS and SPS accelerators as well as the support from the EST division. We would like to thank L. Musa, R. Campagnolo, A. Przybyla, W. Seipp, and B. Windelband for their contribution during construction and commissioning of the TPC and during data taking. We are grateful for excellent support by the CERN IT division for the central data recording and data processing. This work was supported by GSI, Darmstadt, the German BMBF, the German HGF Virtual Institute 146 VI-SIM, the U.S. DoE, the Israeli Science Foundation, the German Israeli and the Minerva Foundations, and the Nella and Leon Benoziyo Center for High Energy Physics Research.

\section{References}

[1] G. Agakichiev et al. (CERES), Phys. Rev. Lett. 75 (1995) 1272 and Eur. Phys. J. C41 (2005) 475.

[2] D. Miskowiec, Nucl. Phys. A774 (2006) 43; A. Marin, J. Phys. G30 (2004) S709; Nucl. Instr. Meth., in preparation.

[3] S. Yurevich, Ph.D. dissertation, Heidelberg University 2006; D. Adamova et al. (CERES), submitted to Phys. Lett. B, nucl-ex/0611022.

[4] M.A. Lisa, S. Pratt, R. Soltz, U. Wiedemann, Ann. Rev. Nucl. Part. Sci. 55 (2005) 357.

[5] D. Adamová et al. (CERES), Nucl. Phys. A714 (2003) 124.

[6] M.A. Lisa et al. (E895), Phys. Lett. B496 (2000) 1.

[7] R.C. Wells, Ph.D. thesis, Ohio State University 2002.

[8] J.Adams et al. (STAR), Phys. Rev. C72 (2005) 014904.

[9] M. Stephanov, K. Rajagopal, and E. Shuryak, Phys. Rev. D 60 (1999) 114028.

[10] H. Sako and H. Appelshäuser, J. Phys. G30 (2004) S1371-1375.

[11] G. Tsiledakis, Ph.D. dissertation, Technical University Darmstadt 2005.

[12] J. Adams et al. (STAR Collaboration), Phys. Rev. C72 (2005) 044902. 